\title{
Factors associated with complications of vaginal hysterectomy in patients with pelvic organ prolapse - a single centre's experience
}

\author{
Nurullah Peker', Edip Aydın², Mustafa Yavuz², Muhammet Hanifi Bademkıran², \\ Serhat Ege ${ }^{2}$, Talip Karaçor ${ }^{3}$, Elif Ağaçayak ${ }^{1}$ \\ ${ }^{1}$ Department of Obstetrics and Gynecology, Dicle University, Faculty of Medicine, Diyarbakır, Turkey \\ ${ }^{2}$ Department of Obstetrics and Gynecology, Health Sciences University, \\ Gazi Yasargil Training and Research Hospital, Diyarbakir, Turkey \\ ${ }^{3}$ Department of Obstetrics and Gynecology, Adıyaman University, Faculty of Medicine, Adıyaman, Turkey
}

\begin{abstract}
Objectives: The study aimed to examine the predisposing factors that play a role in the development of complications in patients undergoing vaginal hysterectomy.

Material and methods: This retrospective analysis was performed on data provided from 239 patients who underwent vaginal hysterectomy due to uterine prolapse at a single centre between January 2008 and August 2018. Complications were defined according to Clavien-Dindo classification of complications. The patients were divided into two groups: with and without complications. We built a model using multivariable logistic regression to examine the relationships between complications and five candidate predictors.

Results: Intra/postoperative complications developed in 30 patients, and the complication rate was found to be $12.5 \%$. $87.2 \%$ of the reported complications were classified as Grade $\leq 2$ according to Clavien-Dindo system. It was found that complications were associated with factors such as intraoperative concurrent salpingo-oophorectomy [Odds ratio (OR): $1.24(1.1-1.4)$ ], low preoperative haemoglobin [OR: 0.96 (0.94-0.98)], uterine weight [OR: 2.69 (2.62-2.76)], and long operation time [OR: $1.04(1.02-1.07)]$. History of pelvic surgery was not found to increase complication rate [OR: 1.11 (0.96-1.27), $p=0.13]$. Our multiple logistic regression model correctly classified $74 \%$ of participants within the Receiver Operating Characteristic (ROC) curve.

Conclusions: Preoperative anaemia, large uterus and concomitant adnexectomy were found to be factors associated with complications during and after vaginal hysterectomy for pelvic organ prolapse.

Key words: vaginal hysterectomy; perioperative complications; predisposing factors; concurrent salpingo-oophorectomy; uterine weight; operation time
\end{abstract}

Ginekologia Polska 2019; 90, 12: 692-698

\section{INTRODUCTION}

Hysterectomy is one of the most widespread gynaecologic operation conducted in the United States, at more than 400,000 per year [1]. A hysterectomy can be completed via many routes depending on the previous history of pelvic surgery, uterine size, degree of descent and experience of the surgeon [2]. Vaginal hysterectomy $(\mathrm{VH})$ is the recommended way of uterus removal in case of benign lesions [3]. Restoration with native tissue or uteruspreserving approaches gained popularity against $\mathrm{VH}$ for pelvic organ prolapse (POP) although it has formerly been one of the most performed methods [4, 5]. Advantages of $\mathrm{VH}$, as compared to abdominal hysterectomies, include shorter hospitalization, more rapid return to normal daily activities and fewer intraoperative and postoperative complications [2, 6]. However, complications such as fever, infection, bleeding, relaparotomy, respiratory and cardiac disorders, re-hospitalisation and death are also seen in $\mathrm{VH}$ as well as in all types of hysterectomy operations [7-9]. These early complications may be associated with various factors, such as age, parity, uterine weight, additional surgeries and length of hospitalisation $[6,7,10]$. 
This study aimed to investigate predisposing factors that play a role in the development of complications in patients with uterine prolapse undergoing $\mathrm{VH}$.

\section{MATERIAL AND METHODS}

The medical records of women who were treated surgically with $\mathrm{VH}$ for uterine prolapse between January 2008 and August 2018 at a tertiary centre were examined retrospectively. For the study, confirmation was provided from the local ethics committee (Approval no. 135).

Medical records of 256 patients who underwent VH within the study period with a main indication of POP were examined. The pelvic organ prolapse quantification (POP-Q) system was used to grade uterine prolapsed before and after the operation (at 1 year) [11]. Patients who had Pessary ring due to high anaesthesia risk or due to her own preference were excluded from the study. Five patients were re-scheduled to the abdominal hysterectomy prior to the operation due to suspicion of deep infiltrative endometriosis $(n=3)$ and inconvenience for lithotomy for hip prosthesis $(n=2)$. Uterine weight was not available for seven patients and therefore those were excluded from statistical analysis.

To standardize surgical outcomes, we have presented a classification of surgical complications according to Clavien-Dindo Classification. This classification is mostly based on the medical results, with major emphasis on the risk and invasiveness of the therapy used to correct a complication.

A total of 239 patients underwent $\mathrm{VH}$ due to pelvic organ prolapse were included. Patients were allocated into two groups: patients with complications (group 1) and patients without complications (group 2). The complications were classified according to Clavien-Dindo classification system [12]. Clavien-Dindo system is one of the most commonly used surgical outcome reporting tools to standardize the adverse outcomes and based on the invasiveness of the therapy and the treatment needed to treat the complication. Grade 1 describes any deflection from the normal postoperative course without any need for intervention. Grade 2 consists of complications that require medical treatment, blood transfusion or parenteral nutrition. Surgical or radiological interventions are classified in Grade 3. Grade 4 consists of life-threatening complications that require intensive care while Grade 5 means death.

Parameters such as age, parity, previous history of surgery, menopause, prolapse stage, presence of additional surgery (colporrhaphy anterior, colporrhaphy posterior, cystorectocele repair, cystorectocele repair plus transobturator tape, cystorectocele repair plus sacrospinous ligament fixation, McCall culdoplasty), presence of concurrent salpingo-oophorectomy, intraoperative complications, duration of the operation, uterine weight in the pathology report, presence of complications in the early postoperative period and the list of complications, if any, whether blood transfusion was performed, preoperative haemoglobin levels, length of hospital stay, and the postoperative day when the Foley catheter was removed were evaluated. After the operation, urinary retention was described as the existence of at least one of the three conditions: 1) first residual urine volume after self-voiding $\geq 150 \mathrm{~mL}, 2$ ) failure of first voiding trial, and 3) Foley catheter re-insertion.

All operations were performed only by specialist doctors whose surgical volume of $\mathrm{VH}$ procedure were at least 20 per year or more. One gr intravenous cefazolin was used for antibiotic prophylaxis. Before the operations, the vulva, vagina and perineum were cleaned. The bladder was emptied with a Foley catheter and the catheter was withdrawn. VH was performed as described by Zimmerman [13]. Salpingectomy or salpingo-ooferectomy was performed where indicated after detailed counselling of the patient by grasping the adnexa with a Babcock clamp and using a Vicryl Endoloop to ease the ligation of the pedicle followed by transection of the adnexa. Apical reinforcement was provided by sacrospinous ligament fixation or McCall Culdoplasty to all patients following VH. Colporrhaphy anterior and/or posterior for prolapses with regard to anterior or posterior compartments, transobturator tape procedure for concomitant clinically proven stress urinary incontinence were performed.

\section{Statistical analysis}

Data were analysed using $\mathrm{R}$ software version 3.5.1 (R Statistical Software, Institute for Statistics and Mathematics, Vienna, Austria). The normal distribution of dataset was confirmed by the Kolmogorov-Smirnov test, and data were presented as mean \pm standard deviation (mean \pm SD). Group comparisons of categorical variables were made using Pearson's chi-square or Fisher exact tests and continuous variables using Mann-Whitney $\mathrm{U}$ and independent samples t-tests.

Complications after VH were evaluated as outcomes for our prediction model. Candidate predictors were determined as salpingo-oophorectomy, pelvic surgery history, preoperative haemoglobin, uterine weight and operation time which are clinically and biologically plausible and associated with complications based on previous studies. Consequently, we included five candidate variables in our final model. Multivariable logistic regression was used to investigate the relationship between outcome and candidate predictors. The model was shown by calculating the area under the curve (AUC). For the variables to be strongly associated with outcomes in the model, ROC curve analysis was built to ease clinical use. The results were evaluated with a $95 \%$ confidence interval $(\mathrm{Cl})$ and a significance level of $\mathrm{p}<0.05$. 


\section{RESULTS}

A total of 256 patients were initially evaluated in this study. The patients not meeting the inclusion criteria were excluded from the study, leaving 239 patients for final evaluation. $\mathrm{VH}+\mathrm{McC}$ all culdoplasty was performed in 12 patients, $\mathrm{VH}+$ Colporrhaphy anterior + + McCall culdoplasty in 27 patients, $\mathrm{VH}+$ Colporrhaphy posterior + McCall culdoplasty in 6 patients, $\mathrm{VH}+$ Colporrhaphy anterior + Colporrhaphy posterior + McCall culdoplasty in 147 patients, VH + Colporrhaphy anterior + Colporrhaphy posterior + transobturator tape + McCall culdoplasty in 26 patients, and VH + Colporrhaphy anterior + Colporrhaphy posterior + Sacrospinous ligament fixation in 21 patients.

Intra/postoperative complications developed in $30 \mathrm{pa}-$ tients, and the complication rate was found to be $12.5 \%$. According to Clavien-Dindo Classification, intraoperative complications occurred in three (1.25\%) patients, whereas postoperative complications were observed in 27 (11.25\%) patients. All three of the intraoperative complications were patients with bladder injuries, for whom primary repair was performed.

Five patients developed febrile morbidity. Ten patients received transfusion of blood products (ranged between one to three erythrocyte suspension and fresh frozen plasma) during the intra/postoperative period. Mild to moderate vaginal bleeding and haemodynamic instability with hypotension and tachycardia were observed in two patients who underwent concurrent salpingo-oophorectomy and subsequently intra-abdominal bleeding was seen in ultrasonographic examination. These patients underwent additional surgery. A laparoscopy procedure could not be performed in these two patients due to the lack of experienced staff for this procedure during at night. Laparotomy was performed on both patients who could not be checked for bleeding through the vaginal route in the lithotomy position under general anestesia. In both patients, bleeding was detected in the infundibulopelvic ligament and brought under control. Pulmonary embolism was seen in one patient on the $20^{\text {th }}$ postoperative day, and the patient was dispatched. Nine patients were re-hospitalised. Haematoma in the vaginal cuff was detected in five of these patients, and improvement was observed after bed rest plus antibiotic treatment. Urinary retention was seen in three (1.2\%) patients, for whom urinary catheterisation was performed. In one patient, the pelvic abscess was detected on the 15th postoperative day, and the abscess was drained by laparotomy under regional anesthesia. No mortality was seen in the patients. $87.2 \%$ of the reported complications were classified as Grade $\leq 2$ according to Clavien-Dindo system. We didn't find any unexpected endometrial pathology (e.g. endometrial carcinoma) after operation in POP patients. The demographic data, clinical features, types of surgery, and developing complications according to Clavien-Dindo Classification criteria of patients are shown in Figure 1.

Comparisons of the patients who developed complications (group 1) and those who did not (group 2) are shown in Table 1. According to the POP-Q staging before and after the operation, all patients' prolapse improved (Tab. 2).

In our model, which we built, four candidate predictors were strongly associated with patients developing complications (Tab. 3). These were salpingo-oophorectomy [OR: $1.24(1.1-1.4)]$, preoperative haemoglobin [OR: 0.96 (0.94-0.98)], uterine weight [OR: 2.69 (2.62-2.76)], and operation time [OR: 1.04 (1.02-1.07)]. The history of pelvic surgery was not associated with patients developing complications [OR: 1.11 (0.96-1.27), $\mathrm{p}=0.13$ ]. Our multiple logistic regression model correctly classified $74 \%$ participants (AIC: 128, AUC: 0.74). The ROC curve plot for candidate predictors is illustrated in Figure 2.

\section{DISCUSSION}

Factors associated with complications in $\mathrm{VH}$ cases were examined in this study. It was found that these complications were associated with uterine weight, intraoperative concurrent salpingo-oophorectomy, low preoperative haemoglobin and extended operation time.

$\mathrm{VH}$ should be the preferred method of hysterectomies, but it is associated with some of the complications. Based on previous studies, the complication rate in $\mathrm{VH}$ varies between 4.1-44.8\% [10]. The complication rate in this study was similar to the literature, and most of the complications occurred during the postoperative period. Relatively higher complication rates in this study might be related to the vigorous data extraction and detailed reporting.

In a systematic review examining perioperative risk factors for $\mathrm{VH}$, concurrent bilateral salpingo-oophorectomy was reported as a risk factor for complications [14]. In another study evaluating salpingo-oophorectomy by the transvaginal route, 127 patients (109 patients with concurrent VH and 18 patients with previous $\mathrm{VH}$ ) had transvaginal salpingo-oophorectomy, and it was found that complications developed in 9 of the 109 patients (fever, bleeding, ureteral injury, return to operating theatre) [15]. In a multi-centre prospective study evaluating 69 patients who underwent bilateral salpingectomy during $\mathrm{VH}, \mathrm{An}$ tosh et al. [16] reported that mean operating time for bilateral salpingectomy was 11 minutes $( \pm 5.6)$, with additional estimated blood loss of $6 \mathrm{~mL}( \pm 16.3)$. There were eight surgical complications, none of which was due to the salpingectomy. In patients evaluated in this study, concurrent salpingo-oophorectomy was not performed in the pre-determined. Instead, it was performed in patients who were found to have easy adnexal access. However, 


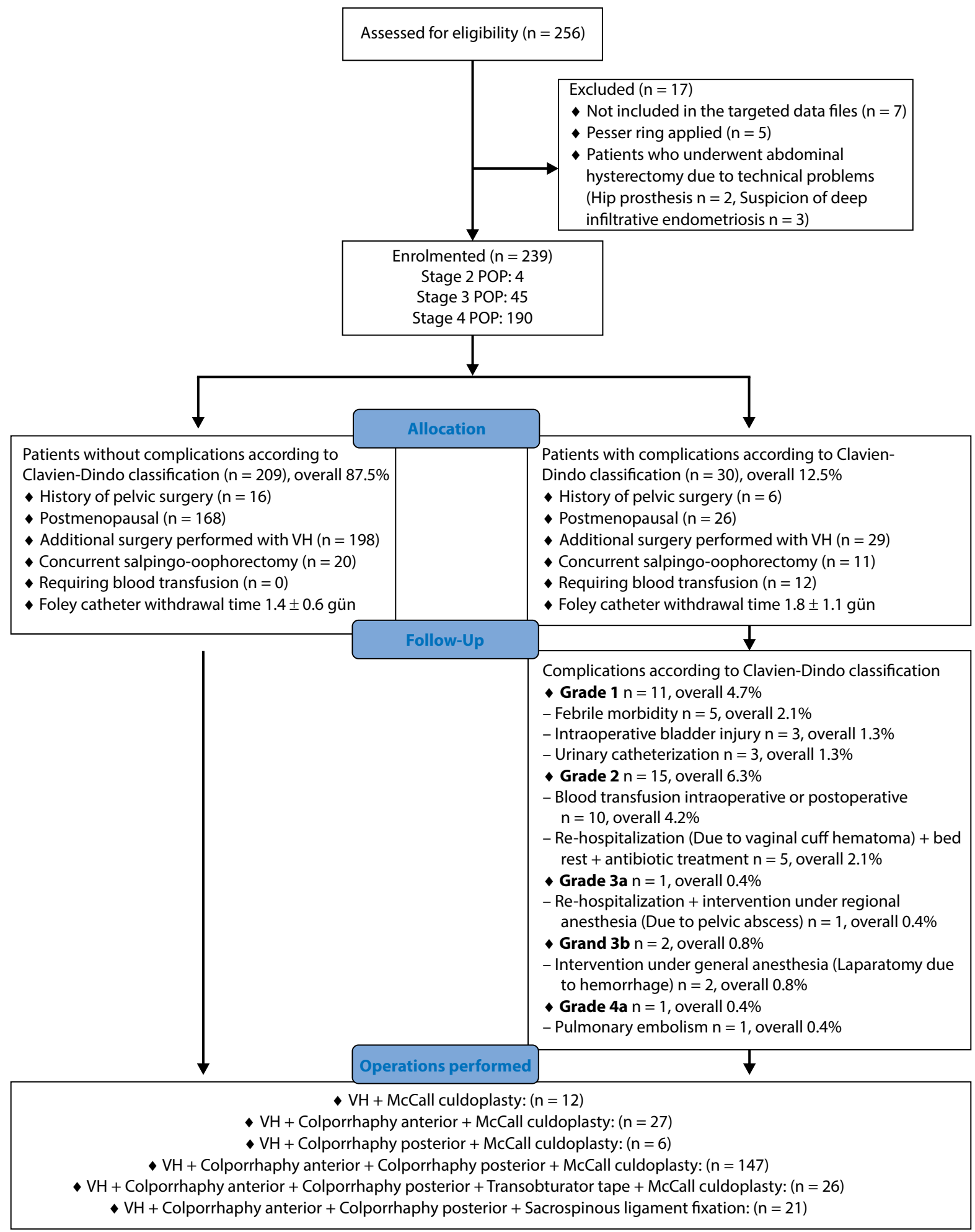

Figure 1. Flow chart of patients

complications were more common in patients who underwent salpingo-oophorectomy.

It is postulated that large uterine size is known to be a cause of technical difficulty in $\mathrm{VH}$. There is no consensus in the literature on the definition of a large uterus regarding weight threshold $[17,18]$. In a retrospective study of 452 cases evaluated by a single surgeon wherein a uterine weight of $250 \mathrm{~g}$ or more was defined as a large uterus, uterine weight and previous vaginal delivery were reported to be a disadvantage for $\mathrm{VH}$ [17]. In another study that 
Table 1. Comparison of patients with and without complications according to Clavien-Dindo classification system

\begin{tabular}{|c|c|c|c|c|}
\hline \multicolumn{2}{|l|}{ Characteristics } & \multirow{2}{*}{$\begin{array}{l}\text { Group } 1 \\
(\mathbf{n}=30) \\
58.2 \pm 9.7 \\
(44-81)\end{array}$} & \multirow{2}{*}{$\begin{array}{l}\text { Group 2 } \\
\text { (n= 209) } \\
60.3 \pm 11.1 \\
(33-83)\end{array}$} & \multirow{2}{*}{$\begin{array}{l}\mathbf{P} \\
0.19\end{array}$} \\
\hline Age [year] & $\begin{array}{l}\text { Mean } \pm S D \\
(\min -\max )\end{array}$ & & & \\
\hline Parity & $\begin{array}{l}\text { Mean } \pm S D \\
(\min -\max )\end{array}$ & $\begin{array}{l}8.5 \pm 2.47 \\
(4-14)\end{array}$ & $\begin{array}{l}7.95 \pm 3.07 \\
(0-16)\end{array}$ & 0.35 \\
\hline Menopausal status & $\begin{array}{l}\text { Yes } \\
\text { No }\end{array}$ & $\begin{array}{l}26(86.7 \%) \\
4(13.3 \%)\end{array}$ & $\begin{array}{l}168(80.4 \%) \\
41(19.6 \%)\end{array}$ & 0.41 \\
\hline History of pelvic surgery & $\begin{array}{l}\text { Yes } \\
\text { No }\end{array}$ & $\begin{array}{l}6(20 \%) \\
24(80 \%)\end{array}$ & $\begin{array}{l}16(7.7 \%) \\
193(92.3 \%)\end{array}$ & 0.04 \\
\hline Concurrent salpingo-oophorectomy & $\begin{array}{l}\text { Yes } \\
\text { No }\end{array}$ & $\begin{array}{l}11(36.7 \%) \\
19(63.3 \%)\end{array}$ & $\begin{array}{l}20(9.6 \%) \\
189(90.4 \%)\end{array}$ & 0.001 \\
\hline Operation time [min] & $\begin{array}{l}\text { Mean } \pm S D \\
(\min -\max )\end{array}$ & $\begin{array}{l}112.1 \pm 29.9 \\
(70-150)\end{array}$ & $\begin{array}{l}82.8 \pm 18.2 \\
(60-150)\end{array}$ & 0.001 \\
\hline Preoperative haemoglobin [g/dL] & $\begin{array}{l}\text { Mean } \pm S D \\
(\min -\max )\end{array}$ & $\begin{array}{l}9.6 \pm 1.8 \\
(6.3-12.8)\end{array}$ & $\begin{array}{l}10.9 \pm 1.1 \\
(8.2-14.3)\end{array}$ & 0.001 \\
\hline Uterine weight [g] & $\begin{array}{l}\text { Mean } \pm S D \\
(\min -\max )\end{array}$ & $\begin{array}{l}244.7 \pm 56.6 \\
(100-320)\end{array}$ & $\begin{array}{l}57.6 \pm 9.4 \\
(40-80)\end{array}$ & 0.001 \\
\hline $\begin{array}{l}\text { Duration of } \\
\text { hospitalization [day] }\end{array}$ & $\begin{array}{l}\text { Mean } \pm S D \\
(\min -\max )\end{array}$ & $\begin{array}{l}5.1 \pm 1.4 \\
(3-10)\end{array}$ & $\begin{array}{l}3.8 \pm 1.2 \\
(2-10)\end{array}$ & 0.001 \\
\hline
\end{tabular}

SD - Standard deviation; min - minimum; max — maximum; Chi-Square Tests; Mann-Whitney U Test; Independent Samples T Test

\begin{tabular}{|l|l|l|l|l|l|}
\hline Table 2. Value of POP-Q before and after operation & \\
\hline & Aa & Ba & Ap & Bp & TVL \\
\hline Preoperative & $2.7 \pm 0.13$ & $2.8 \pm 0.11$ & $2.7 \pm 0.13$ & $2.7 \pm 0.12$ & $6.2 \pm 0.74$ \\
\hline Postoperative & $-2.9 \pm 0.07$ & $-2.89 \pm 0.07$ & $-2.9 \pm 0.06$ & $-2.8 \pm 0.11$ & $-8.5 \pm 0.58$ \\
\hline P value & $\mathrm{p}<0.001$ & $\mathrm{p}<0.001$ & $\mathrm{p}<0.001$ & $\mathrm{p}<0.001$ & $\mathrm{p}<0.001$ \\
\hline
\end{tabular}

Paired-Samples t-test was used

Table 3. Multivariable logistic regression analyses and odds ratios for associations of candidate predictors with complicated vaginal hysterectomy (VH)

\begin{tabular}{|l|l|l|}
\hline Characteristics & OR 95\% CI & p \\
\hline Concurrent salpingo-oophorectomy & $1.24(1.1-1.4)$ & 0.003 \\
\hline History of pelvic surgery & $1.11(0.96-1.27)$ & 0.13 \\
\hline Preoperative haemoglobin & $0.96(0.94-0.98)$ & $<0.001$ \\
\hline Uterine weight & $2.69(2.62-2.76)$ & 0.02 \\
\hline Operation time & $1.04(1.02-1.07)$ & 0.002 \\
\hline
\end{tabular}

$\mathrm{OR}$ - odds ratio; $\mathrm{Cl}$ - confidence interval

evaluated vaginal hysterectomies in women whose uterine weight of $280 \mathrm{~g}$ or more was defined as a large uterus, it was found that large uterine size increased the duration of operation and the amount of bleeding, but there were no differences concerning complications [18]. Another study also reported that $\mathrm{VH}$ could be safely performed with favourable outcomes, even in women with a uterus heavier than $280 \mathrm{~g}$ [19]. In this study, it was determined that average individual uterine weight was higher in the patient

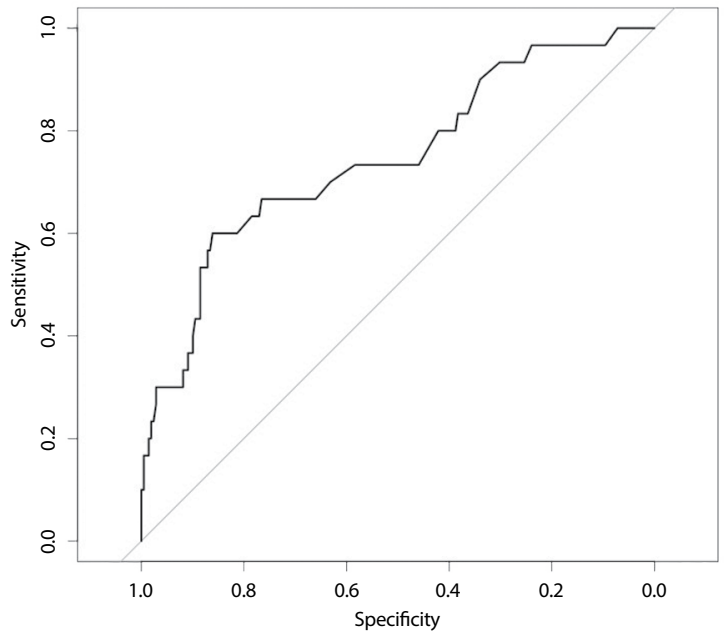

Figure 2. ROC curve plot for candidate predictors

group with complications compared to the group without complications. We believe the reason for this difference could be the fact that the duration of operation was longer 
and the amount of bleeding was higher in patients with a larger uterine size.

One study found that the factors associated with longer operation time were uterine weight, additional surgical operations and estimated blood loss more than $500 \mathrm{~mL}$ [17]. Agostini et al. [20] reported that the complication rate of $\mathrm{VH}$ in nulliparous was higher than in multiparous, and the mean operative time was significantly longer in nulliparous patients. In this study, duration of operation was also found to be significantly higher in patients who developed complications. At the same time, preoperative haemoglobin levels were found to be significantly lower in the group with complications. We are of the opinion that postoperative complications increased in patients with a longer duration of operation due to large uterine size, additional surgeries, such as salpingo-oophorectomy, and increased bleeding. Uterus preserving options may be considered in women who have large uterus and/or scheduled for adnexectomy.

It is controversial whether a history of pelvic surgery is a risk factor for complications in $\mathrm{VH}$. Some studies have argued that a history of previous pelvic surgery is not an obstacle for $\mathrm{VH}$ and does not increase complication rates [19, 21]. Conversely, the study of Akyol et al. [10] and the review of Jeppson et al. [14] reported that a history of previous pelvic surgery was associated with development of complications in $\mathrm{VH}$. In this study, it was found that a history of previous pelvic surgery was statistically higher in the group with complications. However, multivariable logistic regression analysis revealed no risk factor for complications.

In addition to complications, parameters that may affect the length of hospital stay after $\mathrm{VH}$ are significant for discovering the drawbacks of this procedure. One study found that the duration of hospitalisation was $15.6 \pm 7.99$ days for the complicated group and $9.82 \pm 3.97$ days for the uncomplicated group. Patient age, multiparity and presence of complications were found to prolong the duration of hospitalisation [22]. Similar to other studies, it was also noted that hospitalisation was prolonged in patients who developed complications, and those were correlated with the previously mentioned causes.

Previous studies have reported that a decrease in parity and, in particular nulliparity, is associated with complications in $\mathrm{VH}$ [20]. In contrast, in a study evaluating 26 nulliparous vaginal hysterectomies, it was found that the operation failed in only one patient, and there was no difference in complication rates when compared with primiparous and multiparous patients [10]. In the present study, only two patients were nulliparous, for whom VH was completed without complications. There was no significant difference in parity between patients with complications and those without any complications. VH may be technically more difficult in nulliparous com- pared to multiparous women because of vaginal narrowing and less prolapse in nulliparous patients [23]. However, these problems in patients may not pose a disadvantage, depending on the experience of the surgeon [24].

The strengths of our study include a very wide cohort of evaluated cases, in spite of being a single centre study and being the first study to investigate factors associated with complications when cases were classified according to Clavien-Dindo classification system. Besides, the multivariable logistic regression analysis in our model is another factor that makes the study powerful.

We acknowledge the limitations of our data, primarily in that this was a retrospective study, which might be open to selection bias. The same, our study holds data of body mass index, endometriosis, diabetes mellitus, smoking status and uterine pathologies (i.e., myoma uteri). In addition, the fact that the operations were performed by various surgeons may limit the generalizability, however, authors postulate that surgical volume of the operators were well enough with regard to the tertiary nature of the healthcare centre. Lastly, the variables taken into account as the candidate predictors in this study were based on the previous literature. It should be noted that this could cause a limited bias because all patients did not have the same procedures.

In conclusion, although $\mathrm{VH}$ is a safe and traditional method preferred in many patients, it may be associated with various intraoperative and postoperative complications in a certain group of women. Preoperative anaemia, large uterus and concomitant adnexectomy were found to be factors associated with complications during and after $\mathrm{VH}$. Uterus preserving including laparoscopic pexy, colpocleisis or native tissue repair options might be considered in women who has large uterus and/or scheduled for adnexectomy.

\section{Acknowledgements}

We would like to thank Bilge Karaaslan her assistance with the statistics used in this report.

\section{REFERENCES}

1. Wright JD, Herzog TJ, Tsui J, et al. Nationwide trends in the performance of inpatient hysterectomy in the United States. Obstet Gynecol. 2013; 122(2 Pt 1): 233-241, doi: 10.1097/AOG.0b013e318299a6cf, indexed in Pubmed: 23969789.

2. Aarts JWM, Nieboer TE, Johnson N, et al. Surgical approach to hysterectomy for benign gynaecological disease. Cochrane Database Syst Rev. 2015(8): CD003677, doi: 10.1002/14651858.CD003677.pub5, indexed in Pubmed: 26264829.

3. Gupta J. Vaginal hysterectomy is the best minimal access method for hysterectomy. Evid Based Med. 2015; 20(6): 210, doi: 10.1136/ebmed-2015-110300, indexed in Pubmed: 26446039.

4. Boyles SH, Weber AM, Meyn L. Procedures for pelvic organ prolapse in the United States, 1979-1997. Am J Obstet Gynecol. 2003; 188(1): 108-115, doi: 10.1067/mob.2003.101, indexed in Pubmed: 12548203.

5. Yassa M, Tug N. Uterus-preserving Laparoscopic Lateral Suspension with Mesh Operation in Pelvic Organ Prolapse: Initial Experience in a Single Tertiary Center with a Median 24-Month Follow-up. Geburtshilfe Frauenheilkd. 2019; 79(9): 983-992, doi: 10.1055/a-0941-3485, indexed in Pubmed: 31523099. 
6. Pandey D, Sehgal K, Saxena A, et al. An audit of indications, complications, and justification of hysterectomies at a teaching hospital in India. Int J Reprod Med. 2014; 2014: 279273, doi: 10.1155/2014/279273, indexed in Pubmed: 25763395.

7. Louie M, Strassle PD, Moulder JK, et al. Uterine weight and complications after abdominal, laparoscopic, and vaginal hysterectomy. Am J Obstet Gynecol. 2018; 219(5): 480.e1-480.e8, doi: 10.1016/j.ajog.2018.06.015, indexed in Pubmed: 29959931.

8. Dessources K, Hou JY, Tergas Al, et al. Factors associated with 30-day hospital readmission after hysterectomy. Obstet Gynecol. 2015; 125(2): 461-470, doi: 10.1097/AOG.0000000000000623, indexed in Pubmed: 25569007.

9. Karacan T,OzyurekE, Wetherilt LS, et al. Safety and efficacy of using advanced electrosurgical bipolar vessel sealing during vaginal hysterectomy in morbidly obese patients: a retrospective cohort analysis. Ginekol Pol. 2017; 88(10):523-529, doi: 10.5603/GP.a2017.0096, indexed in Pubmed: 29192412.

10. Akyol D, Esinler I, Guven S, et al. Vaginal hysterectomy: results and complications of 886 patients. J Obstet Gynaecol. 2006; 26(8): 777-781, doi: 10.1080/01443610600984529, indexed in Pubmed: 17130029.

11. Persu C, Chapple CR, Cauni V, et al. Pelvic Organ Prolapse Quantification System (POP-Q) - a new era in pelvic prolapse staging. J Med Life. 2011; 4(1): 75-81, indexed in Pubmed: 21505577.

12. Dindo D. The Clavien-Dindo Classification of Surgical Complications. Treatment of Postoperative Complications After Digestive Surgery. 2013: 13-17, doi: 10.1007/978-1-4471-4354-3_3.

13. Zimmerman CW. Vaginal hysterectomy. TeLinde's Oper Gynecol 11 n.d.: P715.

14. Jeppson PC, Balgobin S, Rahn DD, et al. Society of Gynecologic Surgeons Systematic Review Group. Comparison of Vaginal Hysterectomy Techniques and Interventions for Benign Indications: A Systematic Review. Obstet Gynecol. 2017; 129(5): 877-886, doi: 10.1097/AOG.0000000000001995, indexed in Pubmed: 28383375.

15. Sewell T, Courtney H, Tawfeek S, et al. The feasibility and safety of transvaginal bilateral salpingo-oophorectomy. Int J Gynaecol Obstet. 2018; 141(3): 344-348, doi: 10.1002/ijgo.12458, indexed in Pubmed: 29388683.
16. Antosh DD, High R, Brown HW, et al. Feasibility of prophylactic salpingectomy during vaginal hysterectomy. Am J Obstet Gynecol. 2017; 217(5): 605.e1-605.e5, doi: 10.1016/j.ajog.2017.07.017, indexed in Pubmed: 28734829.

17. Sirota I, Tomita S, Dabney L, et al. Overcoming barriers to vaginal hysterectomy: an analysis of perioperative outcomes. J Turk Ger Gynecol Assoc. 2018, doi: 10.4274/jtgga.2018.0021.

18. Newbold $P$, Vithayathil $M$, Fatania $K$, et al. Is vaginal hysterectomy is equally safe for the enlarged and normally sized non-prolapse uterus? A cohort study assessing outcomes. Eur J Obstet Gynecol Reprod Biol. 2015; 185: 74-77, doi: 10.1016/j.ejogrb.2014.11.031, indexed in Pubmed: 25528733.

19. Schmitt JJ, Occhino JA, Weaver $A L$, et al. Outcomes of Vaginal Hysterectomy With and Without Perceived Contraindications to Vaginal Surgery. Female Pelvic Med Reconstr Surg. 2019; 25(1): 41-48, doi: 10.1097/SPV.0000000000000469, indexed in Pubmed: 28914709.

20. Agostini A, Bretelle F, Cravello L, et al. Vaginal hysterectomy in nulliparous women without prolapse: a prospective comparative study. BJOG. 2003; 110(5): 515-518, indexed in Pubmed: 12742338.

21. Chrysostomou A, Djokovic D, EdridgeW, et al. Evidence-based guidelines for vaginal hysterectomy of the International Society for Gynecologic Endoscopy (ISGE). Eur J Obstet Gynecol Reprod Biol. 2018; 231: 262-267, doi: 10.1016/j.ejogrb.2018.10.058, indexed in Pubmed: 30447552.

22. Turgut A, Soydinç HE, Evsen MS, et al. Which parameters may influence the duration of hospitalization after vaginal hysterectomy? J Turk Ger Gynecol Assoc. 2013; 14(1): 15-18, doi: 10.5152/jtgga.2013.04, indexed in Pubmed: 24592064.

23. Ottosen $C$, Lingman $G$, Ottosen L. Three methods for hysterectomy: a randomised, prospective study of short term outcome. BJOG. 2000; 107(11): 1380-1385, doi: 10.1111/j.1471-0528.2000.tb11652.x, indexed in Pubmed: 11117766

24. Mehta A, XuT, Hutfless S, et al. Patient, surgeon, and hospital disparities associated with benign hysterectomy approach and perioperative complications. Am J Obstet Gynecol. 2017; 216(5): 497.e1-497.e10, doi: 10.1016/j.ajog.2016.12.020, indexed in Pubmed: 28034651. 